\title{
Capítulo
}

2

\section{Elementos Fundamentales de Propuestas de Investigación en Interacción Humano Computador}

\author{
Yenny A. Méndez A.
}

\section{Resumen}

El punto de partida de una propuesta de investigación debe estar soportado por la formulación adecuada de los elementos claves que lo conforman, como son la selección del tema, planteamiento del problema y objetivos. Considerando lo anterior, se presenta en este documento una revisión de estos elementos claves, información estrechamente relacionada con la Metodología de la Investigación Holística, incluyendo ejemplos relacionados al área de Interacción Humano Computador.

\subsection{Introducción}

El trabajo que se ha venido realizando en el Centro de Investigación de Estudios Avanzados Sypal ${ }^{1}$ representa un aporte altamente enriquecedor para la formación en investigación y resultado del trabajo que los integrantes realizan, la Dra. Jacqueline Hurtado de Barrera escribió los libros "El proyecto de investigación. Comprensión holística de la investigación y la metodología” [Hurtado 2014a] y “Cómo formular objetivos de investigación. Una comprensión holística” [Hurtado 2014b], que son soporte clave y principal del documento que aquí se presenta.

El contenido de este documento se soporta en una "visión holística”, la cual se relaciona con "una comprensión integradora que trasciende la perspectiva positivista tradicional y abarca los diferentes paradigmas de investigación; la comprensión holística de la investigación integra los diferentes modelos epistémicos y proporciona respuestas y orientaciones para los investigadores de cualquier área del conocimiento humano, incluso en aquellas que tradicionalmente se han considerado vedadas a la investigación científica” [Hurtado 2014a].

1 https://www.cieasypal.com/ 
"Dentro de una comprensión holística la investigación tiene como propósito la búsqueda y generación de conocimiento, de modo tal que este conocimiento pueda tener diferentes grados de elaboración (exploraciones, descripciones, análisis, comparaciones, explicaciones, predicciones, propuestas, transformaciones, verificaciones, evaluaciones)” [Hurtado 2014b].

El proponer una investigación, requiere no solo del planteamiento de los elementos claves que la conforman, sino de la estrecha coherencia entre cada uno de estos elementos. La formulación inicia con una idea o tema de investigación que a partir de lectura de la documentación, se lleva hacia una descripción del problema de investigación, y la formulación de una pregunta de investigación que se buscará resolver con el desarrollo del proyecto. A partir de este planteamiento del problema se especifican los objetivos tanto el general como los específicos, con los que se busca responder a la pregunta de investigación. Posteriormente y dependiendo del tipo de investigación se plantean hipótesis de investigación. Adicionalmente, se requiere incluir una justificación de realizar la investigación.

Se presenta a continuación información de cada uno de los elementos mencionados en el párrafo anterior, relacionando posibles ideas de investigación, que se incluyen con el propósito de clarificar los conceptos y la estructura de los elementos, pero que no pretenden ser en sí mismas propuestas de investigación ya que no se ha realizado un proceso riguroso de revisión de literatura para validar si son novedosas o actuales.

Es de notar que este no es un resultado de investigación ni pretende ser una "camisa de fuerza” en cuanto a lo que se describe, pero si busca que los investigadores y quienes empiecen el camino de la investigación, lo consideren como un soporte para la estructuración de elementos claves de una propuesta de investigación.

\subsection{Elementos fundamentales de una propuesta de proyecto de investigación}

Formular una propuesta de proyecto de investigación requiere plantear elementos, que tienen una estrecha relación. Los elementos claves (Figura 2.1) son: tema, problema a investigar, objetivo general y objetivo específicos, hipótesis (si aplica) y justificación.

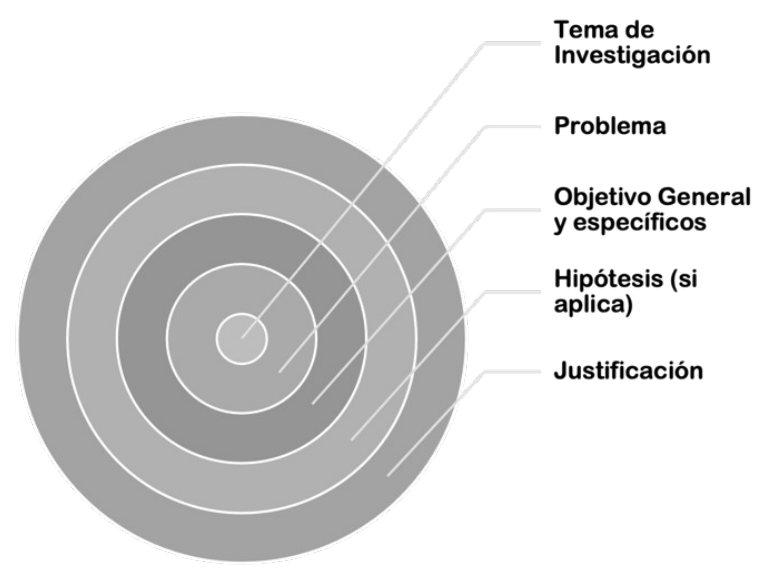


Figura 2.1. Elementos claves de una propuesta de investigación. Fuente: creación propia

De la Figura 2.1 se evidencia que el "punto de partida” es el proponer un tema de investigación, elemento que se presenta en la siguiente sección.

\subsection{Tema de investigación}

El punto de partida es la delimitación del tema en el que hay interés de realizar una investigación, en relación con un área de conocimiento.

La delimitación del tema “corresponde a la fase exploratoria de la investigación, ésta surge cuando el investigador se está planteando la posibilidad de investigar, pero aún no tiene la pregunta de investigación” [Hurtado 2014a]. La selección del tema de investigación puede provenir de diferentes fuentes de información (Figura 2.2).

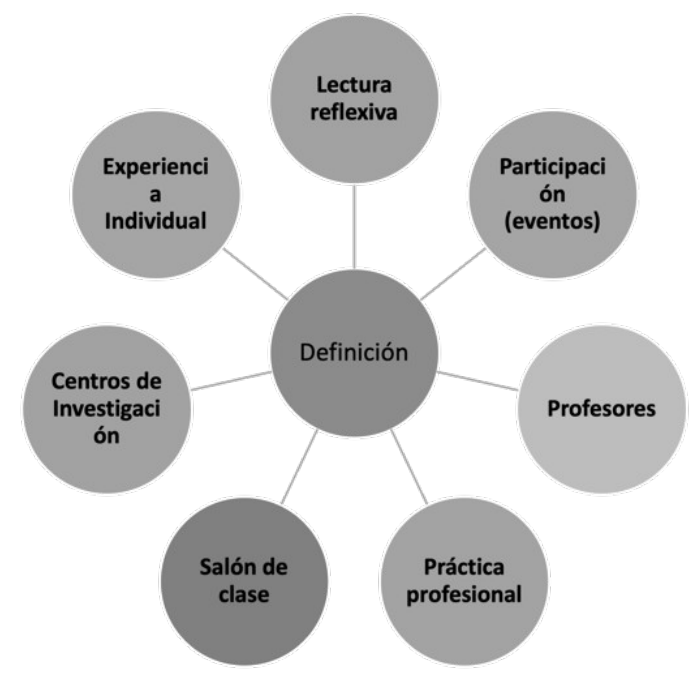

Figura 2.2. Fuentes para identificar ideas de investigación. Fuente: creación propia

Se presentan algunos de los grupos o asociaciones más representativos, en los cuales se pueden identificar investigadores, recursos resultados de investigación y eventos que pueden considerarse como fuentes de información para establecer los temas de investigación a trabajar. Los artículos publicados como resultados de la investigación, presentan información sobre los principales desafíos de IHC y con estos se permite la exploración de temas interesantes, así como temas emergentes publicados en revistas y conferencias.

- Special Interest Group on Computer-Human Interaction (ACM SIGCHI)

El grupo de interés especial ACM sobre Interacción Humano Computador (SIGCHI, por sus siglas en inglés Special Interest Group on Computer-Human Interaction), es "la más grande asociación de profesionales quienes trabajan en la investigación y práctica de HCI”2, de ahí que una fuente adecuada de información es en el contenido que se publica por los diferentes integrantes, así como también la participación de los diferentes eventos de investigación que organiza SIGCHI.

2 https://dl.acm.org/sig/sigchi 
Como parte de SIGCHI, se han conformado capítulos locales que "ayudan a la comunidad ACM SIGCHI a promover y en el avance de la Interacción Humano Computador localmente y alrededor del mundo”3 . Los capítulos organizan conferencias, simposios, reuniones, seminarios, escuelas de verano, foros de discusión y demás espacios de encuentro en relación con la academia y la investigación.

El evento más representativo, organizado por SIGCHI es el CHI: ACM CHI Conference on Human Factors in Computing Systems, en el sitio web ${ }^{4}$ se presenta el listado de conferencias organizadas por ACM SIGCHI.

\section{- Asociación Interacción Persona-Ordenador (AIPO)}

AIPO es "es una asociación profesional abierta a todas las personas interesadas en la Interacción Persona-Ordenador. Sus objetivos son promover y difundir la Interacción Persona-Ordenador y servir de vínculo entre los científicos y profesionales que desarrollen actividades en este ámbito"5.

El evento más representativo, organizado por AIPO es el Congreso Internacional de Interacción Persona-Ordenador ${ }^{6}$, que se viene organizando desde el año 1999. Las actas de los congresos realizados se pueden consultar en el sitio web ${ }^{7}$.

- Red Colaborativa para soportar los procesos de enseñanza-aprendizaje en el área de Interacción Humano - Computador a nivel Iberoamericano (HCI Collab)

La red colaborativa HCI-Collab ${ }^{8}$ es una iniciativa, apalancada por el Dr. César Collazos de la Universidad del Cauca (Colombia) e inicialmente soportada por la Asociación Universitaria Iberoamericana de Postgrado (AUIP), que reúne a académicos e investigadores de diferentes países de Iberoamerica, quienes trabajan sobre el área de Interacción Humano Computador.

El evento más representativo son las Jornadas Iberoamericanas de Interacción Humano Computador, que se vienen realizando en Colombia, México y este año se realizará en Brasil. Adicionalmente, se realiza periódicamente el encuentro "Un año de webinars de IHC en Iberoamérica”, en el que investigadores representativos e integrantes de la red, presentan sus investigaciones. Se encuentra disponible el calendario de webinars del año $2021^{9}$, así como los recursos de soporte de los webinars

\footnotetext{
3 https://sigchi.org/chapters/

4 https://programs.sigchi.org/

5 https://aipo.es/?q=node/1

6 https://aipo.es/?q=congresos

7 https://aipo.es/?q=actas
}

8 https://hci-collab.com/

9 https://hci-collab.com/2021/01/08/wipo2021/ 
ya realizados, recursos que se encuentran del primer año realizado en el $2019^{10} \mathrm{y}$ los recursos del año $2020^{11}$.

Los anteriores grupos alrededor de HCI son representativos y en sus respectivos sitios web se pueden consultar temáticas que son relevantes y actuales, que podrían dar una orientación sobre temas de investigación para trabajar.

En la selección del tema es imprescindible la revisión bibliográfica para tener los criterios suficientes que le permitan establecer si el tema es importante y la necesidad que hay de investigar al respecto. A partir de la revisión se reconocen los trabajos relacionados, lo que se ha consultado sobre el tema, así como los resultados obtenidos [Hurtado 2014].

Algunas preguntas que pueden plantearse al respecto del tema a investigar se presentan en la Figura 2.3.

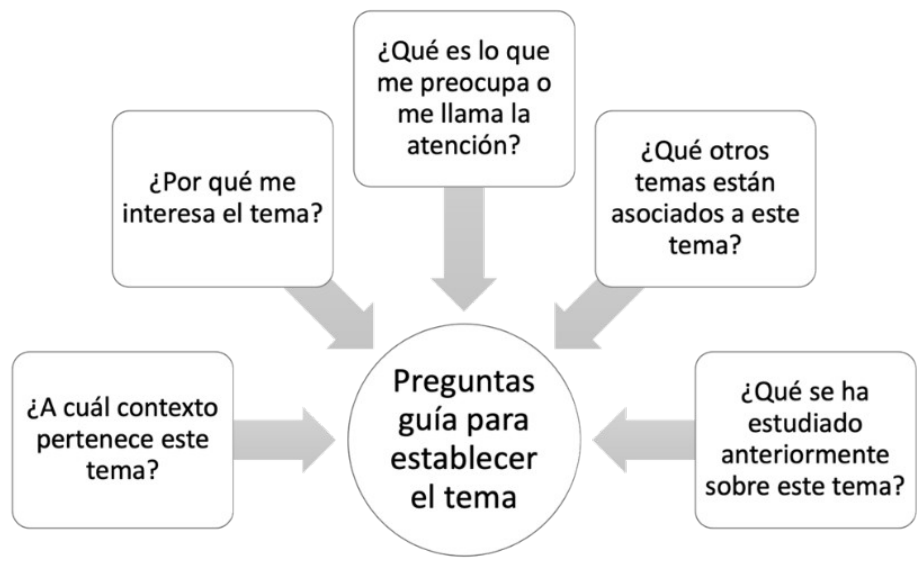

Figura 2.3. Preguntas guía para establecer el tema de investigación. Fuente: Información tomada de [Hurtado 2014a]

Es de notar que algunas de las respuestas a las preguntas planteadas previamente, aportarán a la construcción tanto del problema de investigación como a la justificación. Se recomienda revisar y dar respuesta a cada una de las preguntas para brindar más información sobre el tema de la investigación.

\subsection{Problema de investigación}

Una vez identificado el tema a trabajar en la investigación, se requiere "afinar, precisar y estructurar la idea de investigación” [Sampieri y Mendoza 2018], a través del planteamiento de un problema de investigación.

El planteamiento del problema tiene dos partes esenciales: enunciar el problema y formular el problema, los cuales deben construirse rigurosamente ya que son soporte para la posterior elaboración de los objetivos de investigación [Lerma 2009].

10 https://hci-collab.com/2018/12/20/wipo2019/

11 https://hci-collab.com/2020/01/28/wipo2020/ 
- Enunciar el problema. Tiene relación con mencionar los “antecedentes de estudio y los hechos que originaron las inquietudes e interrogantes iniciales”, "teoría en la que se basó el problema, dejando claro y explícito los vacíos de conocimiento, las inconsistencias encontradas o los puntos que se requieren someter a verificación” [Lerma 2009].

- Formular el problema. A partir de describir la situación problemática se realiza la formulación del problema de investigación en concreto se plantea la pregunta que se va a resolver, esta debe presentar de forma concreta [Lerma 2009].

En la Figura 2.4 y Figura 2.5 se presentan extractos de enunciados de problemas de investigación tomados de artículos de publicados en revistas. El propósito es mostrar ejemplos de información que forma parte de un problema de investigación no entrar al detalle del contenido de los artículos de investigación.

En el contexto chileno, la preocupación por la usabilidad de los sitios de gobierno electrónico ha tenido algunos hitos que se tradujeron en dos instructivos presidenciales, la creación de desarrollo de sitios web, y una evaluación preliminar de TPLs en el año 2005. En Latinoamérica el panorama no es muy distinto. Los estudios son más bien locales y se centran en caracterizaciones de sitios más que en una evaluación de las propiedades de usabilidad de estos (Clancy, 2012). No obstante, no existe un estudio y seguimiento sistemático del estado de la usabilidad de los servicios transaccionales en línea a partir de los cuales tomar medidas para su masificación y explotación de los beneficios derivados de su implementación.

Figura 2.4. Extracto de enunciado de problema de investigación. Fuente: Información tomada de [Palma et. al 2014]

La Organización Mundial de la Salud cifra en 1300 millones, la
cantidad de personas que padecen algún tipo de discapacidad
visual, de las cuales 36 millones son ciegas. La gran mayoría de
personas afectadas por una discapacidad visual se engloban, por lo
tanto, dentro de lo que se considera baja visión. En concreto y, por
lo que respecta a la visión lejana, 188,5 millones de personas
presentan baja visión moderada y 217 millones una visión situada
entre moderada y grave; mientras que por lo que respecta a la
visión de cerca, el número total de personas con baja visión se
estima en unos 826 millones [4]. Una cifra que va de la mano del
aumento del envejecimiento global de la población. En este
sentido, el $86 \%$ de las personas ciegas, el $86 \%$ de las personas con
baja visión y el $61 \%$ de la población con presbicia tienen 50 años
o más [5].

Figura 2.5. Extracto de enunciado de problema de investigación. Fuente: Información tomada de [Alcaráz, et. al 2019]

\subsection{Pregunta de investigación}

La pregunta de investigación, presentada como “enunciado holopráxico” por la Dra. Hurtado (2014), “orienta el estudio hacia único foco, la determinación del alcance del estudio ubica al investigador en el nivel de profundidad en el que desea quedarse". Plantear una pregunta de investigación suele ser complejo y se suelen cometer errores que deberían reconocerse para evitarlos. Se presenta en la Tabla 2.1. listado de errores comúnes al plantear preguntas de investigación. 
Tabla 2.1. Errores comúnes al plantear preguntas de investigación. Fuente: Información tomada de [Hurtado 2014].

\begin{tabular}{|l|l|}
\hline Número & \multicolumn{1}{c|}{ Error } \\
\hline 1 & $\begin{array}{l}\text { Recolectar información sin un propósito definido, después de haber dejado } \\
\text { atrás la fase exploratoria. }\end{array}$ \\
\hline 2 & $\begin{array}{l}\text { Tomar un grupo de datos que ya existen y han sido recogidos por otros } \\
\text { investigadores con propósitos diferentes a la investigación, y tratar de } \\
\text { encajarle una pregunta de investigación. }\end{array}$ \\
\hline 3 & $\begin{array}{l}\text { Definir el enunciado en forma tan ambigua y general que el investigador no } \\
\text { sepa que camino tomar y genere conclusiones arbitrarias. }\end{array}$ \\
\hline 4 & $\begin{array}{l}\text { Plantear una pregunta de investigación sin haber realizado un proceso } \\
\text { previo de exploración y sin haber revisado la bibliografía existente sobre el } \\
\text { tema. }\end{array}$ \\
\hline
\end{tabular}

Se presentan en la Tabla 2.2 a Tabla 2.4, ejemplos de preguntas de investigación que tiene fallas, la razón por las cuales son incorrectos y la posible forma de darle solución.

Tabla 2.2. Ejemplo 1 de formulación pregunta de investigación. Fuente: Creación propia.

\begin{tabular}{|l|l|}
\hline \multicolumn{1}{|c|}{ Aspecto } & \multicolumn{1}{c|}{ Descripción } \\
\hline Pregunta incorrecta & $\begin{array}{l}\text { ¿Cuáles son los problemas de usabilidad que } \\
\text { presentan los sitios web de comercio electrónico } \\
\text { de Santiago de Chile y cómo influyen en las } \\
\text { compras que realizan por internet los usuarios? }\end{array}$ \\
\hline Razón por la que es incorrecta & Involucra dos investigaciones \\
\hline Pregunta correcta & $\begin{array}{l}\text { ¿Cómo influyen los problemas de usabilidad que } \\
\text { presentan los sitios web de comercio electrónico } \\
\text { de Santiago de Chile, en las compras que se } \\
\text { realizan por internet? }\end{array}$ \\
\hline
\end{tabular}

Tabla 2.3. Ejemplo 2 de formulación pregunta de investigación. Fuente: Creación propia.

\begin{tabular}{|l|l|}
\hline \multicolumn{1}{|c|}{ Aspecto } & \multicolumn{1}{c|}{ Descripción } \\
\hline Pregunta incorrecta & $\begin{array}{l}\text { ¿Cuáles son las normativas en España sobre } \\
\text { ergonomía y diseño, que incluyen aspectos a tener } \\
\text { en cuenta para la accesibilidad de las aplicaciones? }\end{array}$ \\
\hline Razón por la que es incorrecta & No conduce a un conocimiento nuevo. \\
\hline Pregunta correcta & $\begin{array}{l}\text { ¿Cuáles son las semejanzas y diferencias que hay } \\
\text { entre las normativas en España sobre ergonomía y } \\
\text { diseño, que incluyen aspectos a tener en cuenta } \\
\text { para la accesibilidad de las aplicaciones y las de } \\
\text { Colombia? }\end{array}$ \\
\hline
\end{tabular}

Tabla 2.4. Ejemplo 3 de formulación pregunta de investigación. Fuente: Creación propia

\begin{tabular}{|l|l|}
\hline \multicolumn{1}{|c|}{ Aspecto } & \multicolumn{1}{c|}{ Descripción } \\
\hline Pregunta incorrecta & $\begin{array}{l}\text { ¿Cuáles son los métodos de evaluación de } \\
\text { usabilidad más utilizados en las empresas de } \\
\text { desarrollo de software? }\end{array}$ \\
\hline Razón por la que es incorrecta & No conduce a un conocimiento nuevo. \\
\hline Pregunta correcta & $\begin{array}{l}\text { ¿Cuáles son los métodos de evaluación de } \\
\text { usabilidad más utilizados en las empresas de } \\
\text { desarrollo de software de la ciudad de Cali } \\
\text { (Colombia) durante los años 2020 y 2021? }\end{array}$ \\
\hline
\end{tabular}


Plantear una pregunta de investigación correcta es clave para enfocar la propuesta de investigación ya que de esta "se derivan los objetivos, tipos de investigación, métodos, los procedimientos y los instrumentos, e incluso se puede tener idea del tipo de conclusiones a las que se pretende llegar”. En una pregunta de investigación "el investigador expresa qué quiere saber, acerca de qué (cuál evento, característica o situación), en quiénes, en cuál contexto y cuándo [Hurtado 2014]. En la Tabla 2.5 se presenta una relación de los aspectos a considerar en una pregunta de investigación (estos aspectos también deben considerarse al plantear objetivos de investigación).

En la Tabla 2.6 a la Tabla 2.11 se presentan ejemplos de relación de interrogantes y respuestas al respecto de preguntas de investigación propuestas.

Tabla 2.5. Aspectos que debe tener una pregunta de investigación. Fuente: Información tomada de [Hurtado 2014a].

\begin{tabular}{|l|l|l|}
\hline \multicolumn{1}{|c|}{ Pregunta } & \multicolumn{1}{|c|}{ Aspecto } & \multicolumn{1}{c|}{ Descripción } \\
\hline $\begin{array}{l}\text { ¿Qué se quiere } \\
\text { saber? }\end{array}$ & $\begin{array}{l}\text { Lo que se desea } \\
\text { saber }\end{array}$ & $\begin{array}{l}\text { Interrogante básico que se presenta } \\
\text { inicialmente al inicio de la pregunta y orienta } \\
\text { hacia el nivel de objetivo a plantear. }\end{array}$ \\
\hline ¿Acerca de qué? & $\begin{array}{l}\text { Evento de } \\
\text { estudio }\end{array}$ & $\begin{array}{l}\text { Característica, hecho, situación, } \\
\text { comportamiento o proceso a estudiar. }\end{array}$ \\
\hline ¿En quiénes? & $\begin{array}{l}\text { Unidad de } \\
\text { estudio }\end{array}$ & $\begin{array}{l}\text { El ser poseedor de la característica a estudiar } \\
\text { o el quien participa del hecho, proceso o } \\
\text { situación. Puede ser una persona, un objeto, } \\
\text { una institución, un país, un grupo, un } \\
\text { documento, un material. }\end{array}$ \\
\hline ¿Cuándo? & Temporalidad & $\begin{array}{l}\text { Lapso en el que se va a estudiar el evento } \\
\text { (presente, pasado, puntual o evolutivo). }\end{array}$ \\
\hline ¿Dónde? & Contexto & $\begin{array}{l}\text { El contexto específico en el cual se va a } \\
\text { estudiar el evento (región geográfica, } \\
\text { institución, ámbito). }\end{array}$ \\
\hline
\end{tabular}

Tabla 2.6. Ejemplo 1 de interrogantes y respuestas en relación con pregunta de investigación. Fuente: creación propia.

\begin{tabular}{|c|c|}
\hline $\begin{array}{l}\text { Pregunta de investi } \\
\text { ingenieros, en temas } \\
\text { software de Colombi }\end{array}$ & $\begin{array}{l}\text { uáles son las necesidades de formación de los } \\
\text { ue han requerido las empresas de desarrollo de } \\
\text { s } 2015 \text { a 2021? }\end{array}$ \\
\hline Pregunta & $\begin{array}{ll}\text { Respuesta } \\
\end{array}$ \\
\hline ¿Qué se quiere saber & ¿Cuáles son...? \\
\hline ¿Acerca de qué? & Necesidades de formación en temas de HCI. \\
\hline ¿En quiénes? & Ingenieros \\
\hline ¿Cuándo? & Años 2015 a 2021 \\
\hline ¿Dónde? & Empresas de desarrollo de software de Colombia \\
\hline
\end{tabular}

Tabla 2.7. Ejemplo 2 de interrogantes y respuestas en relación con pregunta de investigación. Fuente: creación propia.

\begin{tabular}{|c|c|}
\hline $\begin{array}{l}\text { Pregunta de investi } \\
\text { actualmente a los es } \\
\text { Ingeniería en Argent }\end{array}$ & $\begin{array}{l}\text { uáles son las temas que se } \\
\text { n las asignaturas de HCI de } \\
21 \text { ? }\end{array}$ \\
\hline Pregunta & Respuesta \\
\hline ¿Qué se quiere saber & ¿Cuáles son...? \\
\hline ¿Acerca de qué? & Temas que se están impartiendo \\
\hline
\end{tabular}




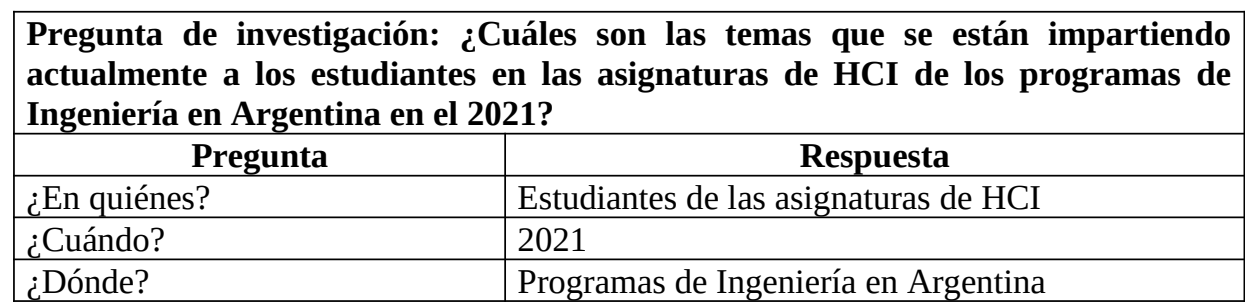

Tabla 2.8. Ejemplo 3 de interrogantes y respuestas en relación con pregunta de investigación. Fuente: creación propia.

\begin{tabular}{|l|l|}
\hline $\begin{array}{l}\text { Pregunta de investigación: ¿En qué medida las competencias que se busca tengan } \\
\text { los estudiantes de las asignaturas de HCI que se imparten en los programas de } \\
\text { pregrado de Ingeniería en México, se corresponden con las competencias que } \\
\text { requieren actualmente tengan los ingenieros de las empresas de software de } \\
\text { México? }\end{array}$ \\
\hline \multicolumn{1}{|c|}{ Pregunta } & \multicolumn{1}{c|}{ Respuesta } \\
\hline ¿Qué se quiere saber & ¿En qué medida...? \\
\hline ¿Acerca de qué? & $\begin{array}{l}\text { Competencias que se busca tengan los estudiantes. } \\
\text { Competencias que requieren actualmente las } \\
\text { empresas }\end{array}$ \\
\hline ¿En quiénes? & $\begin{array}{l}\text { Estudiantes de las asignaturas de HCI. } \\
\text { Ingenieros }\end{array}$ \\
\hline ¿Cuándo? & 2021 \\
\hline ¿Dónde? & $\begin{array}{l}\text { Programas de pregrado de Ingeniería en México. } \\
\text { Empresas de desarrollo de sofware de México. }\end{array}$ \\
\hline
\end{tabular}

\subsection{Objetivos de investigación}

El desarrollo del trabajo de investigación se orienta hacia el logro de los objetivos [Bernal 2010]. Los objetivos son claves en un proceso de investigación ya que son "el punto de arranque para seleccionar, organizar y conducir las acciones a realizar, al tiempo que expresan las metas que orientan la investigación” [Pimienta 2017]. “Los objetivos de investigación están orientados al grado de elaboración de conocimiento que se pretende alcanzar” [Hurtado 2014b].

Tabla 2.9. Ejemplo 4 de interrogantes y respuestas en relación con pregunta de investigación. Fuente: creación propia.

\begin{tabular}{|l|l|}
\hline $\begin{array}{l}\text { Pregunta de investigación: ¿Qué semejanzas y diferencias existen actualmente } \\
\text { entre la calidad del software desarrollado por las empresas de Arequipa (Perú) } \\
\text { que utilizan técnicas Design Thinking y las que hacen uso de técnicas del Diseño } \\
\text { Centrado en el Usuario (D.C.U.)? }\end{array}$ \\
\hline \multicolumn{1}{|c|}{ Pregunta } & \multicolumn{1}{c|}{ Respuesta } \\
\hline$¿$ ¿ué se quiere saber & ¿En qué medida...? \\
\hline ¿Acerca de qué? & Calidad \\
\hline ¿En quiénes? & Software \\
\hline$¿$ Cuándo? & 2021 \\
\hline ¿Dónde? & $\begin{array}{l}\text { Empresas que utilizan técnicas del D.T. de } \\
\text { Arequipa. } \\
\text { Empresas que utilizan técnicas del DCU de } \\
\text { Arequipa. }\end{array}$ \\
\hline
\end{tabular}

Tabla 2.10. Ejemplo 5 de interrogantes y respuestas en relación con pregunta de investigación. Fuente: creación propia. 


\begin{tabular}{|c|c|}
\hline \multicolumn{2}{|c|}{$\begin{array}{l}\text { Pregunta de investigación: ¿Cuáles son las características de los laboratorios de } \\
\text { investigación en HCI para el año 2025, en los grupos de investigación de los } \\
\text { integrantes de la red HCI-Collab?. }\end{array}$} \\
\hline Pregunta & \begin{tabular}{|l|} 
Respuesta \\
\end{tabular} \\
\hline ¿Qué se quiere saber & ¿Cuáles son...? \\
\hline ¿Acerca de qué? & Características \\
\hline ¿En quiénes? & Laboratorios de investigación en HCI \\
\hline ¿Cuándo? & 2025 \\
\hline ¿Dónde? & $\begin{array}{l}\text { Grupos de investigación de los integrantes de la } \\
\text { red HCI-Collab }\end{array}$ \\
\hline
\end{tabular}

Tabla 2.11. Ejemplo 6 de interrogantes y respuestas en relación con pregunta de investigación. Fuente: creación propia.

\begin{tabular}{|l|l|}
\hline $\begin{array}{l}\text { Pregunta de investigación: ¿Qué elementos conformarían un proceso para la } \\
\text { identificación de requerimientos de usabilidad a integrar en una metodología de } \\
\text { desarrollo seguro de software, en las empresas de desarrollo de software de } \\
\text { Santiago de Chile? }\end{array}$ \\
\hline \multicolumn{1}{|c|}{ Pregunta } & \multicolumn{1}{c|}{ Respuesta } \\
\hline ¿Qué se quiere saber & ¿Qué elementos conformarían...? \\
\hline ¿Acerca de qué? & $\begin{array}{l}\text { Un proceso para la identificación de } \\
\text { requerimientos de usabilidad }\end{array}$ \\
\hline ¿En quiénes? & Metodología desarrollo seguro de software \\
\hline$¿$ ¿uándo? & 2021 \\
\hline ¿Dónde? & $\begin{array}{l}\text { Empresas de desarrollo de software de Santiago de } \\
\text { Chile. }\end{array}$ \\
\hline
\end{tabular}

En la investigación holística investigar es: "explorar, describir, analizar, comparar, explicar, predecir, proponer, modificar, confirmar y evaluar” [Hurtado 2014b]

Los objetivos del proyecto se plantean como objetivo general y objetivos específicos. El objetivo general tiene relación con el "planteamiento central que debe llevar a solucionar el problema o tema de investigación” [Pimienta 2017]. Una vez se haya planteado el objetivo general, se requiere generar los objetivos específicos.

Se presentan en la Tabla 2.12 características a considerar en la formulación de objetivos específicos.

Tabla 2.12. Características a considerar al formular objetivos específicos. Fuente: Información tomada de [Pimienta 2017].

\begin{tabular}{|l|l|}
\hline \multicolumn{1}{|c|}{ Característica } & \multicolumn{1}{c|}{ Descripción } \\
\hline Concretos & Que respondan al problema planteado. \\
\hline Realizables & Que se puedan llevar a la práctica. \\
\hline $\begin{array}{l}\text { Enfocados al logro, no a la } \\
\text { actividad }\end{array}$ & $\begin{array}{l}\text { Se requieren realizar actividades para alcanzar los } \\
\text { objetivos, debido a eso se debe revisar con especial } \\
\text { cuidado para que no se planeen las actividades } \\
\text { como objetivos. Actividades como "revisar la } \\
\text { literatura al respecto del tema objeto de estudio" } \\
\text { no deben formar parte de los objetivos específicos. }\end{array}$ \\
\hline
\end{tabular}

El plantear objetivos de investigación "suele ser uno de lo obstáculos que con mayor frecuencia enfrentan los investigadores y particularmente aquellos que se inician en las actividades de indagación”. Si los objetivos de investigación no se plantean de forma adecuada, el principal riesgo es "perderse en el proceso y probablemente termine sin poder dar respuesta a la pregunta que se formuló al inicio” [Hurtado 2014b]. 
Al plantear objetivos de investigación se recomienda dar respuesta a algunas preguntas (Figura 2.6), para validar si realmente es un objetivo de investigación.

Se presentan en la Figura 2.7 ejemplos de objetivos que no corresponden con objetivos de investigación.

La formulación del objetivo general de investigación debe estar directamente relacionada con la pregunta de investigación ya que con el logro del objetivo general se espera dar respuesta a la pregunta. Se presentan algunos ejemplos (Tabla 2.13 a Tabla 2.20) de objetivos generales a partir de una pregunta de investigación.

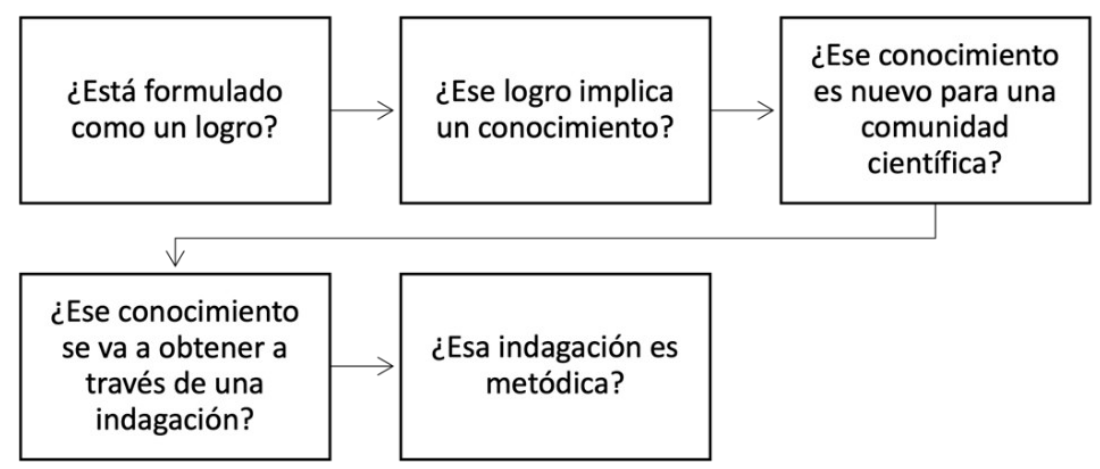

Figura 2.6. Preguntas clave para validar si enunciado corresponde con objetivo de investigación. Fuente: Información tomada de [Hurtado 2014b].

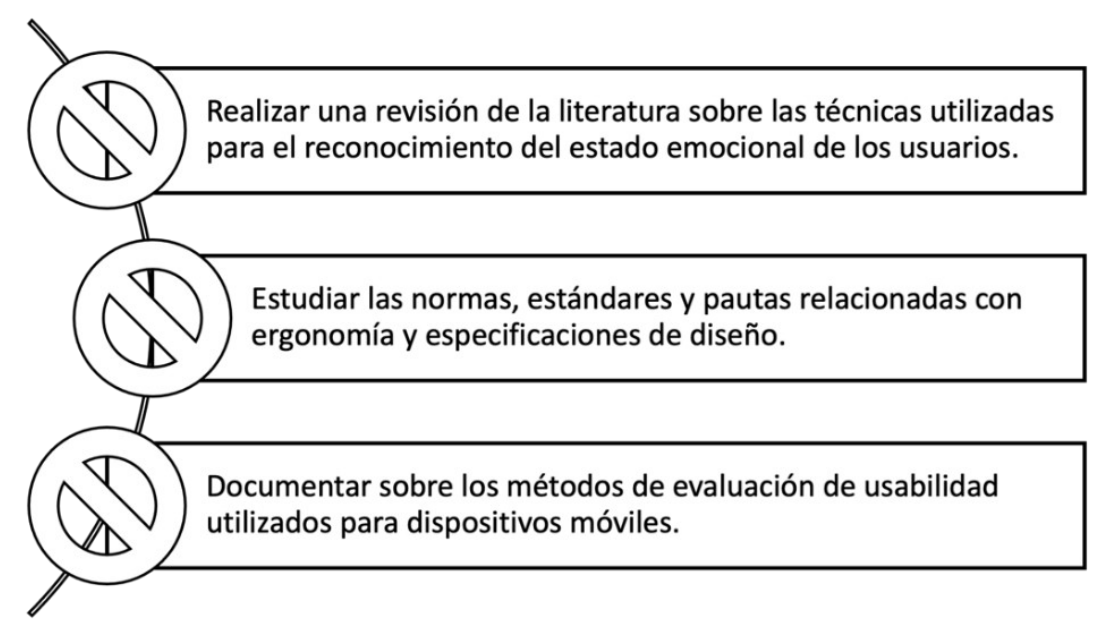

Figura 2.7. Ejemplos de objetivos incorrectos. Fuente: Creación propia

Tabla 2.13. Ejemplo 1 de relación entre pregunta de investigación y objetivo de investigación. Fuente: Creación propia.

\begin{tabular}{|l|l|}
\hline \multicolumn{1}{|c|}{ Aspecto } & \multicolumn{1}{c|}{ Descripción } \\
\hline Pregunta & $\begin{array}{l}\text { ¿Cuáles son las necesidades de formación de los } \\
\text { ingenieros, en temas de HCI que han requerido las } \\
\text { empresas de desarrollo de software de Colombia } \\
\text { en los años 2015 a 2021? }\end{array}$ \\
\hline
\end{tabular}




\begin{tabular}{|l|l|}
\hline \multicolumn{1}{|c|}{ Aspecto } & \multicolumn{1}{c|}{ Descripción } \\
\hline Objetivo incorrecto & $\begin{array}{l}\text { Formular una estrategia didáctica para la } \\
\text { formación de los ingenieros, en temas de que } \\
\text { requieren las empresas de desarrollo de software } \\
\text { de Colombia. }\end{array}$ \\
\hline Razón por la que es incorrecto & $\begin{array}{l}\text { La respuesta a la pregunta de investigación busca } \\
\text { tener información sobre las necesidades de } \\
\text { formación, no está buscando generar alguna } \\
\text { solución. }\end{array}$ \\
\hline Objetivo correcto & $\begin{array}{l}\text { Describir las necesidades de formación de los } \\
\text { ingenieros, en temas de HCI que han requerido las } \\
\text { empresas de desarrollo de software de Colombia } \\
\text { en los años 2015 a 2021. }\end{array}$ \\
\hline
\end{tabular}

Tabla 2.14. Ejemplo 2 de relación entre pregunta de investigación y objetivo de investigación. Fuente: Creación propia

\begin{tabular}{|c|c|}
\hline Aspecto & Descripción \\
\hline Pregunta & $\begin{array}{l}\text { ¿En qué medida las competencias que se busca } \\
\text { tengan los estudiantes de las asignaturas de HCI } \\
\text { que se imparten en los programas de pregrado de } \\
\text { Ingeniería en México, se corresponden con las } \\
\text { competencias que requieren actualmente tengan } \\
\text { los ingenieros de las empresas de software de } \\
\text { México? }\end{array}$ \\
\hline Objetivo incorrecto & $\begin{array}{l}\text { Describir las competencias que se busca tengan los } \\
\text { estudiantes de las asignaturas de HCI que se } \\
\text { imparten en los programas de pregrado de } \\
\text { Ingeniería en México y las competencias que } \\
\text { requieren actualmente tengan los ingenieros de las } \\
\text { empresas de software de México. }\end{array}$ \\
\hline Razón por la que es incorrecto & $\begin{array}{l}\text { Con la investigación se busca comparar las } \\
\text { competencias que tienen los estudianes de las } \\
\text { asignaturas de HCI con las competencias que se } \\
\text { requieren tengan los ingenieros de las empresas. } \\
\text { La descripción podría ser el resultado de un } \\
\text { objetivo específico, pero no el objetivo general de } \\
\text { la investigación. }\end{array}$ \\
\hline Objetivo correcto & $\begin{array}{l}\text { Analizar las competencias que se busca tengan los } \\
\text { estudiantes de las asignaturas de HCI que se } \\
\text { imparten en los programas de pregrado de } \\
\text { Ingeniería en México, en relación con las } \\
\text { competencias que requieren actualmente tengan } \\
\text { los ingenieros de las empresas de software de } \\
\text { México. }\end{array}$ \\
\hline
\end{tabular}

Tabla 2.15. Ejemplo 3 de relación entre pregunta de investigación y objetivo de investigación. Fuente: Creación propia.

\begin{tabular}{|l|l|}
\hline \multicolumn{1}{|c|}{ Aspecto } & \multicolumn{1}{c|}{ Descripción } \\
\hline Pregunta & ¿Qué semejanzas y diferencias existen actualmente \\
& entre la calidad del software desarrollado por las \\
& empresas de Arequipa (Perú) que utilizan técnicas \\
& Design Thinking y las que hacen uso de técnicas \\
& del Diseño Centrado en el Usuario (D.C.U.)? \\
\hline
\end{tabular}




\begin{tabular}{|l|l|}
\hline \multicolumn{1}{|c|}{ Aspecto } & \multicolumn{1}{|c|}{ Descripción } \\
\hline Objetivo incorrecto & $\begin{array}{l}\text { Analizar la calidad del software desarrollado por } \\
\text { las empresas de Arequipa (Perú) que utilizan } \\
\text { técnicas Design Thinking y las que hacen uso de } \\
\text { técnicas del Diseño Centrado en el Usuario }\end{array}$ \\
\hline Razón por la que es incorrecto & $\begin{array}{l}\text { La pregunta de investigación no requiere } \\
\text { únicamente que se haga un análisis al respecto de } \\
\text { la calidad del software ya que requiere se haga una } \\
\text { comparación entre la calidad del software } \\
\text { desarrollado por empresas que hacen uso de las } \\
\text { técnicas del DT y las que hacen uso de las técnicas } \\
\text { del DCU. }\end{array}$ \\
\hline Objetivo correcto & $\begin{array}{l}\text { Comparar el software desarrollado por las } \\
\text { empresas de Arequipa (Perú) que utilizan técnicas } \\
\text { del Design Thinking y las que hacen uso de } \\
\text { técnicas del Diseño Centrado en el Usuario, con } \\
\text { respecto a su calidad. }\end{array}$ \\
\hline
\end{tabular}

Tabla 2.16. Ejemplo 4 de relación entre pregunta de investigación y objetivo de investigación. Fuente: Creación propia

\begin{tabular}{|l|l|}
\hline \multicolumn{1}{|c|}{ Aspecto } & \multicolumn{1}{|c|}{ Descripción } \\
\hline Pregunta & $\begin{array}{l}\text { ¿Qué elementos conformarían un proceso para la } \\
\text { identificación de requerimientos de usabilidad a } \\
\text { integrar en una metodología de desarrollo seguro } \\
\text { de software, en las empresas de desarrollo de } \\
\text { software de Santiago de Chile? }\end{array}$ \\
\hline Objetivo incorrecto & $\begin{array}{l}\text { Evaluar los elementos necesarios de un proceso } \\
\text { para la identificación de requerimientos de } \\
\text { usabilidad a integrar en una metodología de } \\
\text { desarrollo seguro de software, en las empresas de } \\
\text { desarrollo de software de Santiago de Chile. }\end{array}$ \\
\hline Razón por la que es incorrecto & $\begin{array}{l}\text { La respuesta a la pregunta de investigación está } \\
\text { dada para buscar diseñar una propuesta que } \\
\text { contenga los elementos necesarios que } \\
\text { conformarían un proceso para la identificación de } \\
\text { requerimientos de usabilidad, no se llega a la } \\
\text { instancia de realizar una evaluación. }\end{array}$ \\
\hline Objetivo correcto & $\begin{array}{l}\text { Diseñar un proceso para la identificación de } \\
\text { requerimientos de usabilidad a integrar en una } \\
\text { metodología de desarrollo seguro de software, en } \\
\text { las empresas de desarrollo de software de Santiago } \\
\text { de Chile. }\end{array}$ \\
\hline
\end{tabular}

Al igual que la pregunta de investigación, los objetivos deben presentar en su estructura los aspectos presentados previamente en la Tabla 2.5. En concreto, todo objetivo general debe incluir los siguientes aspectos [Hurtado 2014b]:

- Un Verbo en infinitivo que indica “el grado de complejidad del objetivo”.

- El evento o eventos de estudio.

- Las unidades de estudio.

- La temporalidad.

- El contexto. 
En la Tabla 1.17 a la Tabla 1.20 se presenta la identificación de los aspectos en los objetivos presentados como correctos en ejemplos presentados anteriormente.

\section{Tabla 2.17. Aspectos de objetivo específico del Ejemplo 1. Fuente: Creación propia}

\begin{tabular}{|l|l|}
\hline \multicolumn{1}{|c|}{ Aspecto } & \multicolumn{1}{c|}{ Descripción } \\
\hline Objetivo general & $\begin{array}{l}\text { Describir las necesidades de formación de los } \\
\text { ingenieros, en temas de HCI que han requerido las } \\
\text { empresas de desarrollo de software de Colombia } \\
\text { en los años 2015 a 2021. }\end{array}$ \\
\hline Verbo & Describir \\
\hline Evento & Necesidades de formación en temas de HCI \\
\hline Unidades de estudio & Ingenieros \\
\hline Temporalidad & 2015 a 2021 \\
\hline Contexto & Empresas de desarrollo de software de Colombia. \\
\hline
\end{tabular}

Tabla 2.18. Aspectos de objetivo específico del Ejemplo 2. Fuente: Creación propia.

\begin{tabular}{|l|l|}
\hline \multicolumn{1}{|c|}{ Aspecto } & \multicolumn{1}{c|}{ Descripción } \\
\hline Objetivo general & $\begin{array}{l}\text { Analizar las competencias que se busca tengan los } \\
\text { estudiantes de las asignaturas de HCI que se } \\
\text { imparten en los programas de pregrado de } \\
\text { Ingeniería en México, en relación con las } \\
\text { competencias que requieren actualmente tengan } \\
\text { los ingenieros de las empresas de software de } \\
\text { México. }\end{array}$ \\
\hline Verbo & Analizar \\
\hline Evento & Competencias \\
\hline Unidades de estudio & $\begin{array}{l}\text { Estudiantes de las asignaturas de HCI } \\
\text { Empresas de software }\end{array}$ \\
\hline Temporalidad & 2021 \\
\hline Contexto & $\begin{array}{l}\text { Programas de pregrado de Ingeniería en México } \\
\text { Empresas de Software de México }\end{array}$ \\
\hline
\end{tabular}

Tabla 2.19. Aspectos de objetivo específico del Ejemplo 3. Fuente: Creación propia

\begin{tabular}{|l|l|}
\hline \multicolumn{1}{|c|}{ Aspecto } & \multicolumn{1}{c|}{ Descripción } \\
\hline Objetivo general & $\begin{array}{l}\text { Comparar el software desarrollado por las } \\
\text { empresas de Arequipa (Perú) que utilizan técnicas } \\
\text { del Design Thinking y las que hacen uso de } \\
\text { técnicas del Diseño Centrado en el Usuario, con } \\
\text { respecto a su calidad. }\end{array}$ \\
\hline Verbo & Comparar \\
\hline Evento & Calidad \\
\hline Unidades de estudio & Software \\
\hline Temporalidad & 2021 \\
\hline Contexto & $\begin{array}{l}\text { Empresas de Arequipa que utilizan técnicas del DT } \\
\text { Empresas de Arequipa que utilizan técnicas del } \\
\text { DCU. }\end{array}$ \\
\hline
\end{tabular}




\section{Tabla 2.20. Aspectos de objetivo específico del Ejemplo 4. Fuente: Creación propia}

\begin{tabular}{|l|l|}
\hline \multicolumn{1}{|c|}{ Aspecto } & \multicolumn{1}{c|}{ Descripción } \\
\hline Objetivo general & $\begin{array}{l}\text { Diseñar un proceso para la identificación de } \\
\text { requerimientos de usabilidad a integrar en una } \\
\text { metodología de desarrollo seguro de software, en } \\
\text { las empresas de desarrollo de software de Santiago } \\
\text { de Chile. }\end{array}$ \\
\hline Verbo & Diseñar \\
\hline Evento & $\begin{array}{l}\text { Proceso para la identificación de requerimientos de } \\
\text { usabilidad }\end{array}$ \\
\hline Unidades de estudio & Metodología de desarrollo seguro de software \\
\hline Temporalidad & 2021 \\
\hline Contexto & $\begin{array}{l}\text { Empresas de desarrollo de software de Santiago de } \\
\text { Chile. }\end{array}$ \\
\hline
\end{tabular}

En [Hurtado 2014b] se indica que la forma correcta para plantear objetivos de investigación es formulándolo siempre de tal manera que de respuesta a la pregunta de investigación. En la Tabla 1.21 se presenta una la relación propuesta entre preguntas y verbos para el planteamiento de objetivos de investigación.

Tabla 2.21. Relación entre pregunta y verbos de objetivos de investigación. Fuente: Información tomada de [Hurtado 2014b].

\begin{tabular}{|l|l|}
\hline \multicolumn{1}{|c|}{ Pregunta } & \multicolumn{1}{c|}{ Verbo } \\
\hline ¿Qué hay & Explorar \\
\hline $\begin{array}{l}\text { ¿Cómo es...? ¿Cuántos...? } \\
¿ \text { Cuáles...? }\end{array}$ & Describir \\
\hline $\begin{array}{l}\text { ¿En qué medida se } \\
\text { corresponde...? }\end{array}$ & Analizar \\
\hline ¿Qué diferencia hay...? & Comparar \\
\hline ¿Por qué...? & Explicar \\
\hline ¿Cómo será en el futuro...? & Predecir \\
\hline ¿Cómo sería una propuesta...? & Proponer \\
\hline $\begin{array}{l}\text { ¿Qué cambios se pueden } \\
\text { producir...? }\end{array}$ & Modificar \\
\hline ¿Qué relación existe...? & Confirmar \\
\hline ¿Qué tan efectivo es...? & \\
\hline
\end{tabular}

$\mathrm{Al}$ respecto de los objetivos específicos, es de notar que "los estadios por los que debe pasar la investigación se expresan en los objetivos específicos” [Hurtado 2014b].

\subsection{Hipótesis de investigación}

Las hipótesis de investigación se plantear en aquellas investigaciones en las que se requiere probar el impacto que se tiene entre las variables o el efecto de una variable sobre otra. "La hipótesis es una posible respuesta a cualquier problema que nos planteemos, aunque todavía no sepamos si es cierta o no” (Pimienta, De la Hoz, 2017).

"La hipótesis es un enunciado que propone una respuesta tentativa a la pregunta del problema de investigación. Tiene carácter de tentativa porque la relación que se 
proponga sobre las variables debe ser sometida a comprobación empírica. Esta comprobación empírica hace que la hipótesis orienta la selección del diseño del estudio, la recolección y el análisis de los datos hacia ser aceptada o no” (Lerma, 2009).

\subsection{Justificación}

La justificación “comprende argumentaciones y razonamientos que permiten justificar la elección de esa investigación, en ese contexto, con estas unidades de estudio, en ese momento" [Hurtado 2014a].

La justificación de realizar la investigación se debe soportar en su importancia, para lo cual se debe considerar (Lerma, 2009):

- Que los resultados sean utilizados por alguien.

- La necesidad de aplicar, crear o aclarar algún aspecto teórico sobre determinada parte del objetivo de la investigación.

- Que los resultados proporcionen aporte metodológico sobre algún proceso.

- Los resultados sean necesarios para tomar una decisión inmediata.

- Que los resultados de la investigación estén de acuerdo con las prioridades de la empresa, localidad, región o país.

Es necesario justificar cada uno de los aspectos que forman parte de una pregunta de investigación, de los presentados en la Tabla 2.5. Se presenta en la Tabla 2.22 información sobre los aspectos de la pregunta de investigación.

Tabla 2.22. Aspectos de la pregunta de investigación y descripción de contenido a incluir en justificación. Fuente: Información tomada de [Hurtado 2014b].

\begin{tabular}{|l|l|}
\hline \multicolumn{1}{|c|}{ Pregunta } & \multicolumn{1}{c|}{ Verbo } \\
\hline Evento de estudio & $\begin{array}{l}\text { Justificar la razón por la cual se seleccionó el } \\
\text { evento de estudio, en remplazo de otros posibles } \\
\text { evntos. }\end{array}$ \\
\hline Unidades de estudio & $\begin{array}{l}\text { Justificar la razón por la cual se seleccionó la } \\
\text { unidad de estudio entre diferentes unidades. }\end{array}$ \\
\hline Contexto & $\begin{array}{l}\text { Presentar información de por qué la investigación } \\
\text { debe realizarse en el contexto seleccionado. }\end{array}$ \\
\hline Temporalidad & $\begin{array}{l}\text { Justificar la selección del momento en el que se } \\
\text { decide realizar la investigación. }\end{array}$ \\
\hline
\end{tabular}

\section{Consideraciones finales}

El hacer investigación no está soportado en una receta que de el paso a paso de forma concreta. Sin embargo, se deben considerar las recomendaciones y hallazgos que han tenido los investigadores a partir de sus estudios, de sus experiencias e incluso de sus propios errores.

Es altamente requerido plantear los elementos que integran una propuesta de investigación de tal forma que exista una coherencia entre cada uno de estos. El plantear un tema, problema de investigación, pregunta de investigación y objetivos sin un factor común, hará que el investigador no logre nunca llegar a los resultados que se planteó llegar. 
Una vez definidos los elementos fundamentales de la propuesta de investigación, se tiene una base sólida para continuar procedimientos para el logro de los objetivos, los cuales son [Hurtado 2014a]:

- $\quad$ Determinación del tipo de investigación.

- $\quad$ Selección del diseño de investigación.

- $\quad$ Definición de eventos o fenómenos estudiados, así como de los indicios de medición.

- Delimitación, selección y descripción de las unidades de estudio (población y muestra).

- $\quad$ Selección de técnicas e instrumentos de recolección de datos.

- $\quad$ Descripción del procedimiento.

- $\quad$ Selección de las técnicas de análisis de resultados.

Los procedimientos presentados anteriormente se escapan del propósito del presente documento, sin embargo, se recomienda la lectura de [Hurtado 2014a] para profundizar en cada uno de los procedimientos y en general en la profundización de los aspectos mencionados en el documento.

Se desea que el contenido del presente documento sea una base para el fortalecimiento de las propuestas de investigación en temáticas relacionadas con Interacción Humano Computador y en general temáticas para hacer investigación. El aporte principal fue el de incluir ejemplos que permitan su reflexión en el ámbito de IHC y dar a conocer el significativo trabajo que se viene realizando en Centro de Investigación de Estudios Avanzados Sypal, sobre procesos y material que orienten en la investigación a las diferentes áreas del conocimiento.

\section{Referencias}

Alcaraz, R., Ribera, M. and Granollers, T. (2019). "Gráficos estadísticos accesibles para personas con baja visión y visión cromática deficient”. En: Congreso Internacional de Interacción Persona-Ordenador. http://hdl.handle.net/10459.1/71518.

Bernal, C. (2010). “Metodología de la investigación”. Administración, economía, humanidades y ciencias sociales. Prentice Hall.

Hernández-Sampieri, R. and Mendoza, C. (2018). "Metodología de la investigación: Las rutas cuantitativa, cualitativa y mixta”. México. McGraw Hill Education.

Hurtado, J. (2014). "El proyecto de investigación. Comprensión holística de la metodología y la investigación”. (7 $7^{\mathrm{a}}$ ed.). Ediciones Quirón S.A.

Hurtado, J. (2014). “Cómo formular objetivos de Investigación”. Magisterio Editorial.

Lerma González, H. D. (2009). Metodología de la investigación: propuesta, anteproyecto y proyecto. Ecoe Ediciones.

Palma, M. G., Tapia, C. L. and Rodríguez-Peña, N. (2014) "Medición De Usabilidad De Trámites Públicos En Línea En Chile: Un Caso De Estudio En Gobierno Electrónico/Usability Measure of Chilean Public Online Services: an E-Government 
Case Study". Journal of Information Systems and Technology Management: JISTEM, 11(1), 85-104. https://doi.org/10.1590/S1807-17752014000100006

Pimienta, J. y De la Orden, A. (2017). "Metodología de Investigación”. México. Pearson Educación. 